\title{
Exploring the motion advantage: evaluating the contribution of familiarity and differences in facial motion
}

\section{Natalie Butcher \& Karen Lander}

To cite this article: Natalie Butcher \& Karen Lander (2016): Exploring the motion advantage: evaluating the contribution of familiarity and differences in facial motion, The Quarterly Journal of Experimental Psychology, DOI: 10.1080/17470218.2016.1138974

To link to this article: http://dx.doi.org/10.1080/17470218.2016.1138974

Accepted author version posted online: 28 Jan 2016.

Submit your article to this journal $\sqsubset$

à

View related articles $[\pi$

View Crossmark data $־$ 
Motion Characteristics and Familiarity

Publisher: Taylor \& Francis \& The Experimental Psychology Society

Journal: The Quarterly Journal of Experimental Psychology

DOI: $10.1080 / 17470218.2016 .1138974$

Exploring the motion advantage: evaluating the contribution of familiarity and differences in facial motion

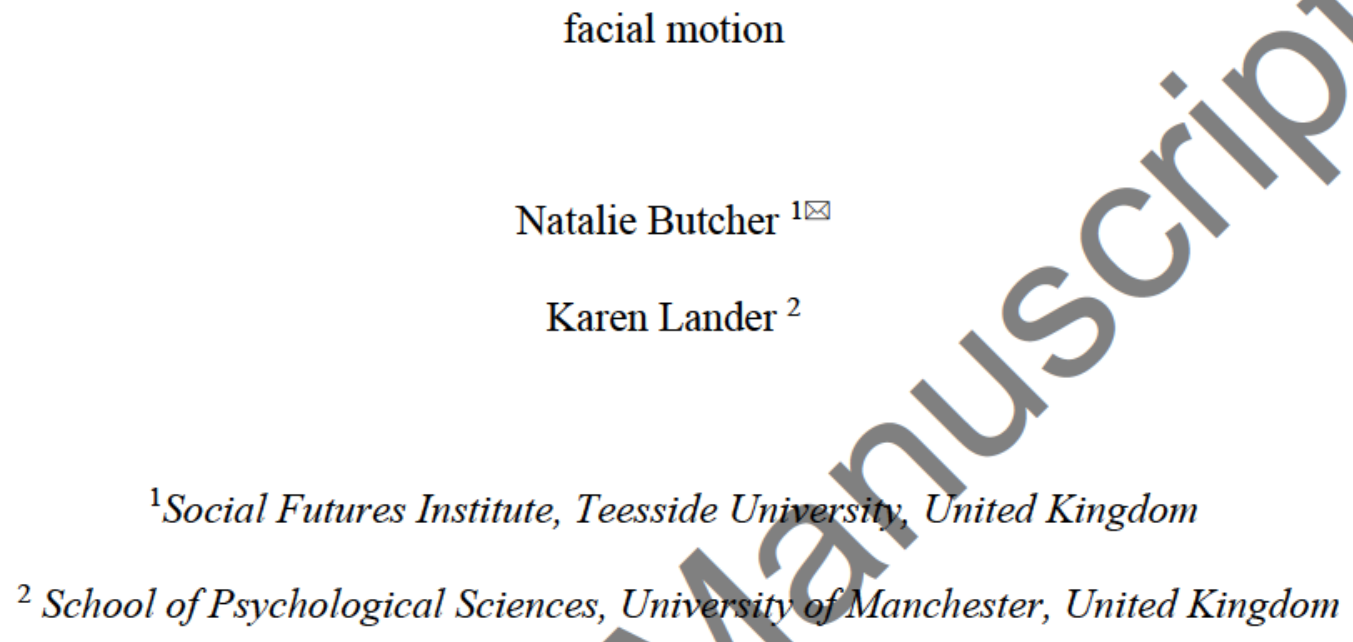

Correspondence concerning this article should be addressed to: Dr Natalie Butcher, Teesside University, Social Futures Institute, Middlesbrough, TS13BA $\square$ : n.butcher@tees.ac.uk

Word count (excluding abstract, title page, references): 6107

Number of figures: 1

Acknowledgement: This work was supported by an EPSRC/BBSRC grant (reference: EP/D056942/1). The authors have no financial interest or benefit from the direct application of their research and the research was conducted in accordance with BPS ethical guidance with approval from the School of Psychological Sciences Ethics Committee, University of Manchester. 


\section{Abstract}

Seeing a face move can improve familiar face recognition, face matching and learning. More specifically, familiarity with a face may facilitate the learning of an individual's 'dynamic facial signature'. In the outlined research we examine the relationship between participant ratings of familiarity, the distinctiveness of motion, the amount of facial motion and the recognition of familiar moving faces (Experiment 1) as well as the magnitude of the motion advantage (Experiment 2). Significant positive correlations were found between all factors. Findings suggest that faces rated as moving a lot and in a distinctive manner benefited the most from being seen in motion. Additionally findings indicate that facial motion information becomes a more important cue to recognition the more familiar a face is, suggesting that 'dynamic facial signatures' continue to be learnt over time and integrated within the face representation. Results are discussed in relation to theoretical explanations of the moving face advantage.

Key words: Face identity, facial motion, familiarity, distinctiveness, recognition

\section{Introduction}

There is much evidence that seeing a face move optimises learning and recognition (for a review see Xiao et al., 2014). Indeed, facial motion has been demonstrated to lead to better learning of previously unfamiliar faces (Butcher, Lander, Fang \& Costen, 2011; Lander \& Bruce, 2003; Pike, Kemp, Towell, \& Philips, 1997); more accurate and faster face matching (Thornton \& Kourtzi, 2002); and better identification of degraded familiar faces (Knight \& Johnston, 1997; Lander, Bruce \& Hill, 2001). This effect is referred to as the 'motion 
Motion Characteristics and Familiarity

advantage' (e.g., Knight \& Johnston, 1997; Lander, Christie, \& Bruce, 1999; Lander \& Davies, 2007; O’Toole, Roark, \& Abdi, 2002; Pike et al., 1997; Schiff, Banka \& De Bordes Galdi, 1986) and it is thought that facial motion plays a supplementary role to static facial information in identity recognition (Roark, Barrett, Spence, Abdi \& O'Toole, 2003). Whilst the motion advantage is robust in studies investigating the recognition of familiar faces (e.g. Knight \& Johnston, 1997; Lander, Christie \& Bruce, 1999, Lander et al., 2001-Lander \& Chuang, 2005), research investigating the role of facial motion in the learning of previously unfamiliar faces has been less consistent (see Bruce et al., 1999, Bruce, Henderson, Newman \& Burton, 2001; Christie \& Bruce, 1998). Pike et al. (1997) found beneficial effects of facial motion, as faces seen rotating rigidly were better recognised than those learnt as multiple static images. On the other hand, using a similar incidental learning task, Christie and Bruce (1998) found no advantage of studying previously unfamiliar faces in motion or testing memory for faces in motion. Thus, face familiarity may be a key factor in understanding the role facial motion plays in identity recognition and learning.

Importantly two, non-mutually exclusive, mechanisms have been proposed to explain how facial motion facilitates identity recognition and learning (O'Toole et al., 2002; Roark, et al., 2003). These differing mechanisms may help explain why the motion advantage is more robust for familiar faces than unfamiliar faces (Bennetts et al., 2013). Unfamiliar faces can benefit from the first mechanism; the representation enhancement hypothesis (O'Toole et al., 2002), which suggests that facial motion aids recognition by facilitating the perception of the three-dimensional structure of the face. It posits that the quality of the structural information available from a human face is enhanced by facial motion and, importantly, this benefit surpasses that provided by seeing the face from many static viewpoints (Christie \& Bruce, 1998; Lander et al., 1999; Pike et al., 1997). Recent work by Butcher et al. (2011) supports this hypothesis by showing that it is more important that a face is learnt in motion, than 
recognized from a moving clip. Here, facial motion may help build a more robust face representation by providing enhanced structural information at learning. Importantly this mechanism is not dependent on any previous experience with a face, and thus may help us understand how motion aids the learning of previously unfamiliar faces (O'Toole et al., 2002; but see recent work by Bennetts et al., 2013).

In addition to the structure from motion role, a second mechanism, the supplemental information hypothesis (O'Toole et al., 2002) assumes that we represent the characteristic facial motions of an individual's face as part of our stored facial representation.Characteristic motions are those that are idiosyncratic of a particular individual in terms of the spatiotemporal dynamics of the motion (Knappmeyer, Thornton \& Bülthoff, 2003). Using computer animation techniques Knappmeyer, Thonton and Bülthoff (2003) provide unmistakable support for the supplemental information hypothesis (O'Toole et al., 2002). In their experiment participants viewed and thus became familiar with either, head A exhibiting motion from volunteer A, or head B exhibiting motion from volunteer B. In the test phase an animated head constructed from the morph of the two synthetic heads (A and B) was produced. Participants were asked to identify whose head was shown. It was found that participant's identity judgements were biased by the motion they had originally learnt from head A or B, indicating that the human face recognition system integrates individual nonrigid facial motion with facial form information during identity processing. For the particular individual's 'characteristic motion signatures' to be learnt and become intrinsic to that person's facial representation, experience with the face is needed (O'Toole et al., 2002; Roark, et al., 2003). Familiarity is therefore inherent within this explanation of the motion advantage. Whilst much research has investigated the effect of familiarity on identity recognition in general (e.g. Burton, Wilson, Cowan \& Bruce, 1999; Ellis, Shepherd \& Davies, 1979; Young, Hay, McWeeny, Flude \& Ellis, 1985) limited work has investigated the 
more specific relationship between face familiarity and the motion advantage. Based on the supplemental information hypothesis (O'Toole et al., 2002) we would expect that the more familiar we are with a face and its motion, the more useful motion becomes as a cue to identity. Partial support for this idea comes from Roark, O'Toole, Abdi and Barrett (2006) who found an increasing role for motion with familiarity, but this effect was only present in the face-to-gait condition (Experiment 1). Conversely, Lander and Davies (2007) found that the beneficial effect of motion is not dependent on the amount of time a face is viewed and Bennetts et al., (2013) found no evidence that familiarity with a face leads to a larger motion advantage. Here, participants were shown famous or unfamiliar faces and were asked to match from a non-degraded image to a point-light display or shape normalised avatar. Additionally, Bruce et al. (2001) conducted a series of experiments investigating whether familiarity with a face influences the presence and magnitude of the motion advantage. Whilst they found that familiarity aided recognition overall, no motion advantage was found for unfamiliar or personally familiar faces (Experiment 1). In Experiment 2, Bruce et al. (2001) varied the number of times viewers saw a 30s moving clip of a face and found that viewing each face twice did not improve recognition performance over a single viewing. These findings suggest that recognition performance and the magnitude of the motion advantage do not increase as a function of increased familiarity with a face and its motion. However, these studies have simply compared 'known' or 'unknown' faces (Bruce et al., 2001, Experiment 1; Bennetts et al., 2013) or have focused on experimentally familiar faces (Bruce et al., 2001, Experiment 2, 30s to 60s exposures; Lander \& Davies, 2007, 30 minutes to 2 hours; Roark et al., 2006, 9s to 36s) as opposed to prolonged real world familiarity that reflects the non-dichotomous nature of familiarity. It may be that familiarity effects are based on more than relatively brief exposure to items and that brief exposure is not sufficient to determine whether a particular pattern of facial movement is characteristic of that person. 
Indeed, the relationship between the motion advantage and familiarity as a result of realworld exposure of varying levels, including extensive experience is yet to be fully understood.

Further consideration of the beneficial effect of face motion reveals that familiarity may not be the only factor that contributes to the magnitude of the motion advantage gained. Research indicates that disruptions to the natural movement of the face can influence the size of the motion advantage. Lander et al. (1999) and Lander and Bruce (2000) found lower recognition rates for famous faces when facial motion was slowed down, speeded up, reversed or rhythmically disrupted. Furthermore Lander, Chuang and Wickham (2006) used a morphing technique to create intermediate face images between the first and last frames of a natural smile. When shown in sequence, these images were used to create an artificially moving smile that lasted the same amount of time and had the same start and end point as the natural smile for that individual. They found that recognition was better when faces were shown naturally smiling compared to a static neutral face, a static smiling face or a morphed smiling sequence. This finding is consistent with the predictions of the supplemental information hypothesis (OToole et al., 2002), as access to characteristic motion signatures is presumably disrupted when the viewed facial motion is not consistent with the stored characteristic motion signature, i.e. with its natural tempo and rhythm. These behavioural studies were supported by Schultz, Brockhaus, Bülthoff and Pilz (2013) who found that activation in the superior temporal sulcus (STS) was stronger when facial movements appeared more fluid. The posterior superior temporal sulcus (pSTS) is argued to be involved in the processing of dynamic facial information such as head rotation, eye gaze and facial expression as well as the extraction of motion information from invariant face aspects such as face identity (Bernstein \& Yovel, 2015). Taken together these findings demonstrate that seeing the precise dynamic characteristics of the face in motion provides the greatest 
advantage for facial recognition. Furthermore it is possible that the type of motion displayed by a face (e.g. rigid or non-rigid) might contribute to the presence of the motion advantage (Roark et al., 2003) and provide at least a partial account for inconsistencies in the literature, for example when different types of rigid movements are displayed (e.g. Christie \& Bruce, 1998; Pike et al., 1997). There is however limited research investigating the relationship between differences in facial motion displayed naturally (as opposed to artificial manipulations to motion characteristics) and the motion advantage. For static face recognition a clear benefit for faces that are thought to be spatially distinctive has been revealed, as findings indicate that distinctive faces are better recognised than faces that are rated as being 'typical' (Bartlett, Hurry, \& Thorley, 1984; Light, Kayra-Stuart, \& Hollander, 1979; Valentine \& Bruce, 1986; Valentine \& Ferrara, 1991, Vokey \& Read, 1992). Recent research has also revealed better recognition of faces previously paired with distinctive voices compared to typical voices suggesting that facial distinctiveness is multisensory (Bülthoff \& Newell, 2015). It is therefore worth considering whether distinctiveness of facial motion might also influence face recognition and be related to the magnitude of the motion advantage gained by a face. Only one experiment has previously directly investigated this issue: Lander and Chuang (2005) found that the more distinctive or characteristic a person's motion was rated to be, the more useful a cue to recognition motion was, demonstrating that differences in the motion displayed by a face can moderate the motion advantage. However, this finding is yet to be replicated and other differences in facial motion (e.g. how much a face moves) have to date been ignored.

Here we aim to investigate the relationship between familiarity, the distinctiveness of motion, amount of facial motion and recognition of moving faces (Experiment 1) and the magnitude of the motion advantage (Experiment 2). We aim to provide a greater understanding of the motion advantage by indicating whether differential effect sizes for 
Motion Characteristics and Familiarity

different stimuli may, in part, be related to differences in the motion displayed by any given face and an observers familiarity with that face.

\section{Experiment 1}

In this experiment participants were asked to recognise moving famous faces and rate the same faces for familiarity, how much facial motion was exhibited and distinctiveness of facial motion. If the motion advantage for familiar faces can, at least in part, be explained by characteristic motion signatures of a face being stored in memory and learnt over time (supplemental information hypothesis; O'Toole et al., 2002) then there should be a positive relationship between recognition and familiarity. Recognition of the moving famous faces will be greater for faces participants are familiar with i.e. when characteristic motion information for that individual has been learnt and integrated into the representation of that face. In addition, face recognition may be better for faces that are rated to move more and move more distinctively.

\section{Method}

Design. A correlational design was used to measure the relationship between the four variables of interest; recognition rates (the number of times each face was correctly recognised was measured by participant's vocal responses), rated face familiarity, perceived distinctiveness of motion and amount of facial motion (each measured on 10-point likert scales).

Participants. Fifty participants (46 female) aged between 19 and 21 (mean age 19 years and 10 months) participated in the study. All were students from the University of Manchester 
Motion Characteristics and Familiarity

paid in participation credits. All had normal or corrected to normal eyesight and had not taken part in any studies of this kind previously.

Stimuli and apparatus. 58 grey scale moving clips were selected from a bank of video sequences of famous faces previously used for facial recognition experiments (see Lander \& Bruce, 2000; Lander et al., 1999, 2001). The famous faces included actors and other TV personalities, sportsmen and women, members of the royal family and politicians. All displayed at least the head and shoulders of the person. As the images were originally taken from television footage some were seen from the waist upwards Motion displayed during the clips was mainly non-rigid (movements in which individual parts of the face move in relation to one another, for instance, during speech and expression) but there was some rigid motion of the head and rotation about the waist. Clip duration was edited to $2 \mathrm{~s}$ and clips were displayed negated during the recognition task, to reduce recognition rates below ceiling levels (Galper, 1970; Johnston, Hill, Carman, 1992; Kemp, Pike, White, \& Musselman, 1996; Liu \& Chaudhuri, 1998; Luria \& Strauss, 1978; Philips, 1972). Negation converts the pattern of brightness (Russell, Sinha, Biederman \& Nedderhouser, 2006), preserving edge information whilst removing other cues to identity such as pigmentation and shape, determined from shading processes that inform the observer as to the 3D structure of a face (Bruce \& Young, 1998; Cavanagh \& Leclerc, 1989; Hill \& Bruce, 1996; Johnston et al., 1992; Kemp et al., 1996). However, negation maintains the availability of motion information. Stimuli were presented on a G4 PowerMac using Psyscope Software (Cohen, McWhinney, Flatt \& Provost, 1993). All the movies were presented in the centre of a $40.6 \mathrm{~cm} \times 30.5 \mathrm{~cm}$ Mitsubishi, Diamond Plus 230 screen and were $9 \mathrm{~cm} \times 6 \mathrm{~cm}$ in size $(320 \times 240$ pixels $)$. However, the size of each face on the screen varied in width (between $1.6 \mathrm{~cm}$ and $4.5 \mathrm{~cm}$ ) due to the nature of the footage. 
Procedure. Experiment 1 consisted of a recognition task and a rating task. The recognition task was conducted first, in which participants were presented with the 58 famous face stimuli, one at a time in a set pseudo-random order to allow the experimenter to note verbal responses accurately. After each 2 s moving clip was presented, an 8-second inter-stimulus interval (ISI) took place during which the participants informed the experimenter who the face just seen belonged to. Responses were deemed correct if the participant was able to provide the name of the person or some other specific semantic information abøut the person. Names of characters played or programmes people had acted in (e.g., "Fox Moulder" or "The X-files' for David Duchovny) were deemed as correct recognitions, as were unambiguous descriptions of the person. General information such as "comedian", "politician" or "actor" without support of further information about the person were not sufficient to be regarded as correct recognition.

Once all 58 famous faces had been shown participants were given the opportunity to take a short break followed by the rating task. In the rating task participants were presented with the same 58 famous face movie clips (this time without negation) and asked, following each clip, to rate the face on three parameters; how familiar the person was to them, how much facial motion they perceived the face to display during the clip and how distinctive they believed the motion displayed by each face to be. These three questions were presented in the same order after each stimulus. Each factor was measured using a likert scale of 0-9 with 0 being the least and 9 being the largest amount of each of the parameters. Participants were told to complete this task at their own pace using the number keys on the main key pad of the keyboard.

\section{Results and discussion}


Analysis presented throughout this paper is by-item rather than by-participant in order to investigate the relationship between recognition of a specific face, the facial motion it displays and familiarity. The mean correct recognition rate across faces was $40.34 \%$ (SD = 29.26) demonstrating variation in recognition rates across faces. The mean rating for each rated factor was; familiarity, $7.75(\mathrm{SD}=1.35)$, amount of motion, $4.62(\mathrm{SD}=1.30)$ and distinctiveness of motion, $4.85(\mathrm{SD}=1.70)$ (see figure 1.). A Kolmogorov-Smimnov test of normality revealed that the rating data was not normally distributed. Therefore Spearman's Rho correlations were conducted to investigate the relationship between each rated parameter and recognition rates and the Benjamini-Hochberg's correction method (Benjamini \& Hochberg, 1995) was used to control false discovery rate (FDR). Recognition rates were found to be significantly correlated with each of the rated parameters; familiarity, $r^{s}(56)=$ $.69, p<.001$, amount of facial motion, $\mathrm{r}^{\mathrm{s}}(56)=35, p<.01$ and distinctiveness of facial motion, $\mathrm{r}^{\mathrm{s}}(56)=.52, p<.001$ such that recognition rates increased as ratings of each of these factors increased. As would be expected participants were better at recognising faces that they were more familiar with This could be argued to be indicative of face representations becoming stronger and as a result more unique and easier to differentiate with increased experience. Interestingly if was also found that differences in the motion displayed by a face were also related to recognition. Highly recognisable faces were perceived to move relatively more than others and perceived to move more distinctively relative to other faces. Whilst previous research has found that spatially distinctive faces are better recognised than faces that are rated as being 'typical' (Bartlett, Hurry, \& Thorley, 1984; Light, Kayra-Stuart, \& Hollander, 1979; Valentine \& Bruce, 1986; Valentine \& Ferrara, 1991; Vokey \& Read, 1992) this is only the second study, to our knowledge, to demonstrate that faces displaying highly distinctive motion are better recognised (Lander \& Chuang, 2005) and the finding that the amount of motion a face displays is related to recognition rates is new and has not been 
demonstrated previously. Additionally significant positive correlations were found between; familiarity and how much motion was displayed, $\mathrm{r}^{\mathrm{s}}(56)=.49, p<.001$; familiarity and distinctiveness of motion, $\mathrm{r}^{\mathrm{s}}(56)=.71, p<.001$, as well as how much motion was seen and how distinctive the motion was perceived to be, $\mathrm{r}^{\mathrm{s}}(56)=.80, p<.001$. The current findings therefore indicate that perceived distinctiveness of facial motion and amount of motion displayed increase as familiarity increases. In Experiment 2 we explore whether these differences in motion characteristics are also related to the size of the motion advantage

[Figure 1 about here]

\section{Experiment 2}

We have already shown in Experiment 1 that there is a positive relationship between familiarity and recognition rates for moving famous faces, as was to be expected. Interestingly, we also found a relationship between differences in facial motion displayed by each face and recognition rates. It is therefore possible that differences in motion characteristiesmight be related to the extent to which a face benefits from being seen in motion at recognition i.e. the magnitude of the motion advantage. In Experiment 2 we tested this hypothesis directly. Using a simple recognition task Experiment 2 aimed to replicate the findings of Lander et al., (1999) comparing levels of familiar face recognition from a single static image with recognition from a two second moving clip to establish overall whether a motion advantage exists (Knight \& Johnston, 1997; Lander, et al., 1999). Secondly by combining the recognition results of this study with the motion parameter ratings collected in Experiment 1 we investigated whether the extent to which a face benefits from being seen in 
motion is related to how familiar the face is, how much it is perceived to move and how distinctively it moves. If the motion advantage is related to differences in the motion displayed by a face we would expect to find a relationship between the motion advantage gained by a particular face and the characteristics (amount and distinctiveness) of the motion it displays.

\section{Method}

Design. A within-participants experimental design was used manipulating the independent variable of presentation style; static or moving. The dependent yariable was recognition rates for each face (as in Experiment 1). Correlational analyses were also conducted to investigate the relationship between the magnitude of the motion advantage and the three variables of interest rated in Experiment 1 (face familiarity, perceived distinctiveness of motion and amount of facial motion). Ratings from Experiment 1 were utilised here to control for any influence of conducting the recognition taskin the same session as the rating task.

Participants. Twenty participants (12 female) with a mean age 19 years and 11 months participated in this study, All were students from the University of Manchester paid in participation eredits. All had normal or corrected to normal eyesight and had not taken part in any experiments of this kind previously.

Materials. The same 58, grey scale, negated moving clips used in Experiment 1 were used again here for the moving condition. For each of the 58 famous face stimuli used in Experiment 1 a still image was created from the original 2 s movie clip for use in the moving condition. The static image of each face was chosen so that it represented a typical image of that person, in that it did not display an unusual facial expression or pose. Both the moving 
clips and still images were $9 \mathrm{~cm} \times 6 \mathrm{~cm}$ (320 x 240 pixels) in size but as in Experiment 1 the size of each face within the image ranged from $1.6 \mathrm{~cm}$ for the smallest to $4.5 \mathrm{~cm}$ for the largest face. The stimuli were presented in the centre of the $40.6 \mathrm{~cm} \mathrm{X} 30.5 \mathrm{~cm}$ screen on a G4 PowerMac using Psyscope software (Cohen et al., 1993).

Procedure. The experiment involved a recognition task split into two blocks. In one block participants saw 29 faces in static and in the other block participants viewed the remaining 29 faces in motion so that each participant was presented with all 58 face stimuli but they viewed half of the faces in motion and the other half in static. Block order and the faces used in the static / moving conditions was counterbalanced across participants. Each face was presented individually in a set pseudo-random order for 2 s. After each moving clip / static image was presented there was a 8s ISI, during which the screen was blank. Participants were then asked to indicate to the experimenter whether they recognised the person they had seen. Responses were deemed correct using the same criteria employed in Experiment 1. Participants were given no feedback. After the participant had responded to the first 29 faces they were given the opportunity to take a short break following which they then viewed the second 29 faces.

\section{Results and discussion}

Across all items the mean recognition was $37.33 \%(\mathrm{SD}=27.53)$ with a mean recognition rate of $26.72 \%(\mathrm{SD}=26.52)$ in the static presentation condition and $47.93 \%(\mathrm{SD}$ $=31.39$ ) in the moving presentation condition. In order to address concerns that the findings in Experiment 1 may have been the result of participants completing both the recognition and rating task in the same session an initial analysis sought to replicate the findings from Experiment 1 by investigating the correlations between Experiment 1 ratings and the 
recognition performance of participants in Experiment 2's motion condition. As discussed previously a Kolmogorov-Smirnov test of normality revealed that overall the ratings data collected in Experiment 1 were not normally distributed. As a result Spearman's rho correlations were again conducted and the Benjamini-Hochberg's correction method (Benjamini \& Hochberg, 1995) was used to control false discovery rate (FDR) across all correlations conducted in Experiment 2. As in Experiment 1, recognition rates for moving faces were found to be significantly correlated with each of the rated parameters; familiarity, $\mathrm{r}^{\mathrm{s}}(56)=.63, p<.001$, amount of facial motion, $\mathrm{r}^{\mathrm{s}}(56)=.30, p<.05$ and distinctiveness of facial motion, $\mathrm{r}^{\mathrm{s}}(56)=.49, p<.001$ such that recognition rates increased as ratings of each of these factors increased. These findings serve to confirm the findings from Experiment 1 and reduce, to some extent, concerns regarding the recognition task and rating task being completed within the same experimental session in Experiment 1 as here the findings have been replicated with different participants taking part in the two tasks.

A paired sample t-test was then conducted to investigate whether a motion advantage was present. The t-test revealed, as hypothesized, that when faces were viewed as a moving clip participants recognised them significantly better than when the famous face was to be recognised from a static image, $t(57)=8.71, p<0.01, d=1.14, \mathrm{CI}[-2.61,-1.63]$. Therefore since a motion advantage was established here it was now possible to explore whether the magnitude of the motion advantage faces received was related to familiarity and each of the motion parameters rated in Experiment 1; distinctiveness of motion and amount of motion. Motion advantage was calculated by computing the difference between the number of correct recognitions in the motion condition compared to the static condition. The mean motion advantage was $2.12(\mathrm{SD}=1.85)$. It was found that the magnitude of the motion advantage was significantly correlated with familiarity, $\mathrm{r}^{\mathrm{s}}(56)=.22, p<0.05$ suggesting, in line with the 
supplemental information hypothesis (O'Toole et al., 2002), that facial motion becomes a more important cue to recognition identity the more familiar the face is. It is therefore suggested that characteristic motion information continues to be learnt over time, becoming intrinsic to that particular face representation. Additionally it was found that the magnitude of the motion advantage was also significantly correlated with amount of motion displayed $\mathrm{r}^{\mathrm{s}}(56)=.23, p<0.05$, and motion distinctiveness, $\mathrm{r}^{\mathrm{s}}(56)=.24, p<0.05$. Therefore, faces that were perceived to be moving a lot and displaying highly distinctive motion benefifed most from being seen in motion when compared to recognition from a single static image. This finding indicates that differences in facial motion may be related to the motion advantage and lead to some faces benefitting more from the availability of motion information relative to others, that do not move a lot or in a distinctive manner,

\section{General Discussion}

In two Experiments, we investigated whether familiarity with a face and differences in facial motion, specifically amount and distinctiveness of motion, are related to recognition of moving famous faces (Experiment 1) and the magnitude of the motion advantage (Experiment 2). In addition, we replicated the motion advantage previously seen for familiar faces presented in non-optimal viewing conditions (Knight \& Johnston, 1997; Lander et al., 1999) demonstrating how robust the phenomenon is. However, our two main findings also reveal the complexity of the motion advantage. First, recognition of moving familiar faces and the magnitude of the motion advantage were both found to be related to face familiarity. Second, differences in the motion displayed by the to-be-recognised face was also found to be related to recognition of moving familiar faces and the size of the motion advantage derived by each face.

Interestingly, we found an overall effect of familiarity in Experiment 1, as it was found that famous faces were better recognised the more familiar they were to the observer. This is in 
line with previous studies that have found familiarity to help face matching when previously unfamiliar and familiar faces are compared (Bennetts et al., 2013; Bruce et al., 2001). Moreover, in Experiment 2 we found, in line with Roark et al. (2006) that a relationship does exist between familiarity and the magnitude of the motion advantage. This finding differs to previous research which has failed to find a relationship between face familiarity and the magnitude of the motion advantage, but importantly these earlier studies compared 'known' and 'unknown' or experimentally familiar faces (Bennetts et al., 2013, Bruce et al., 2001; Lander \& Davies, 2007) rather than measuring the variance in familiarity levels acquired through prolonged real world exposure. Here then the magnitude of the motion advantage was related to overall familiarity with that face. This finding supports the predictions of the supplemental information hypothesis (O'Toole et al., 2002) and provides, at least apartial, account for the robust nature of the motion advantage for familiar faces compared to unfamiliar faces. It suggests that, although characteristic facial motion patterns can be leamt rapidly (Lander \& Davies, 2007) this supplementary identity specific information becomes more useful to identity processing with time and experience as we continue to become more familiar with both the form and motion of the face. This finding demonstrates that motion information is useful to the recognition of a face to varying degrees dependent on how familiar the face is. However, familiarity ratings for each face were collapsed across participants and whilst familiarity in Experiment 1 was conceptualised as the level of personal familiarity, in Experiment 2 ratings of familiarity were not provided by the same participants who undertook the recognition task so here familiarity was conceptualised as overall familiarity (i.e. how well known they are to the public in general), so it remains to be seen whether individual participant familiarity with a face influences this relationship between face familiarity and the magnitude of the motion advantage. It is likely however that the relationship would be enhanced for faces we are personally more familiar with, which might partially explain the more modest correlation seen in Experiment 2 between the 
motion advantage and familiarity. However it is likely that the modesty of the correlations in Experiment 2 relating to the motion advantage are modest due to the nature of the motion advantage measure used and the limited variance in scores on this measure compared to the variance seen in recognition rates.

Additionally the experiments presented here established that differences in facial motion displayed by a face, namely the amount and distinctiveness of motion, are related to how easily recognisable that face is and the extent to which it benefits from being viewed in motion. In line with Lander and Chuang (2005) we successfully replicated, for the first time, the finding that the more distinctive a person's motion was rated to be, the more useful a cue to recognition motion was. This finding also corroborates Bulthoff and Newell's (2015) suggestion that the nature of facial distinctiveness is not limited to static cues; instead it is multisensory integrating static, motion and vocal based information. It is possible that familiarity with a face might lead to that face's motion being perceived as distinctive relative to less familiar faces because the motion is familiar to the viewer and it is this distinctiveness which makes the face easier to differentiate from others However, we must remember that distinctiveness of motion may not only refer to a motion that is characteristic or typical of a particular individual but also to motions that are odd for a particular individual to produce, as well as any motion that is generally odd or unusual. Whilst cause and effect cannot be established here, results from Experiment 1 nay be indicative that familiarity with a face leads to a more distinctive representation of that face's characteristic motion as ratings of familiarity and distinctiveness of motion were found to be significantly correlated. However, when considering explanations of the correlation between familiarity and distinctiveness of motion we cannot rule out the impact of the second form of distinctiveness; motions that are odd for a particular individual to produce could also lead to a correlation with familiarity. That is, for us to know that the movement is odd 
for that particular individual, we must already have experience with and be familiar with the motions they typically display.

The finding that recognition of moving faces and the magnitude of the motion advantage is related to the amount of motion a face is perceived to display is particularly interesting. This finding is new and to our knowledge has not been demonstrated previously. One possible explanation for this finding is that as a face becomes more familiar we increasingly attend to its motion, having already stored the basic structural information. This is a reasonable hypothesis given previous research that has demonstrated encoding of the three-dimensional shape of a face to be important when we originally build a face representation (Bonner, Burton \& Bruce, 2003; Bruce et al., 1999). This notion is also supported by the positive correlation found in Experiment 1 between ratings of familiarity and ratings of how much motion was perceived. As familiarity increased participants were significantly more likely to perceive the face as moving more. The relationship between familiarity and amount of motion may be crucial to our understanding of the motion advantage so future research should investigate whether perceptions of amount of motion are moderated by familiarity. Alternatively, it is possible that faces were rated as moving more because they moved distinctively, causing the motion to be more noticeable to the observer. Again the significant correlation between ratings of motion distinctiveness and amount of motion offers some support for this explanation. However, using our participant ratings of amount of facial motion (Experiment 1) Cristinacce and Cootes (2008) found that participant ratings of amount of motion correlated well with an automatic computer estimate of the amount of facial motion displayed in the moving clips we used, $\mathrm{r}^{\mathrm{s}}(57)=.68, p<.01$ suggesting that the most plausible explanation of this finding is that faces that move more derive a greater motion advantage. This motion characteristic therefore warrants further investigation along with systematic analysis of kinematic variables e.g. velocity, as a recognition advantage for faces that move more is implied by the current finding. 
It is also possible that faces that moved a lot or in a distinctive manner may have attracted more attention from the participants and this heightened attention may have led to the improvement in recognition rather than the motion characteristics per se. This notion is in line with the social signals explanation (O'Toole et al., 2002) of the motion advantage, which posits that the social cues carried in movement may attract attention to the identity specific areas of the face, facilitating identity processing. Therefore future research should endeavour to investigate the underlying mechanism causing the relationship between these differences in facial motion and recognition performance.

A possible limitation of the experiments presented here is that ratings of the motion characteristics and familiarity may have been influenced by pre-exposure to the faces in the recognition task (Experiment 1). It is therefore possible that the faces rated here as moving a lot and moving distinctively are not typically high in facial motion or motion distinctiveness so whether the ratings are reflective of the specific clips or stored representations (activated by the recognition task) of how much a face typically moves remains to be seen. It will be important for future investigations to collect motion parameter ratings in a session that does not involve a recognition task so that recognition memory can be minimised as a possible influence on the way participants rated the faces in Experiment 1.

The results of the current study have two major implications for our understanding of the motion advantage in face recognition. First, that differences regarding how a face moves might be related to how easily recognisable the face is and the extent to which it gains from being seen in motion. Future experimental work needs to investigate whether the differences in facial motion ratings are indicative of characteristic motion or are clip dependent. This can be done by exploring whether ratings of amount and distinctiveness of motion are stable over different clips of the same face. If ratings are consistent across multiple clips and the relationship with recognition performance and motion advantage remains this would suggest, in line with the 
Motion Characteristics and Familiarity

supplemental information hypothesis (O'Toole et al., 2002) that, for efficient recognition to be achieved, characteristic motion information, including the amount and distinctiveness of motion, is stored in our mental representation of familiar faces and accessed at recognition to provide a supplemental cue to identity when this dimension of identity specific information is useful to recognition over and above the information provided by static cues. Second, we found that that the magnitude of the motion advantage was related to how familiar the face was-Moreover these findings support a flexible usage hypothesis such that both task demands (Morrison \& Schyns, 2001; Oliva \& Schyns, 1997; Schyns \& Oliva, 1999) and individual differences factors, including familiarity, amount of facial motion and distinctiveness of motion effect the usefulness of facial motion information during the recognition process.

\section{References}

Bartlett, J. C., Hurry, S., \& Thorley, W. (1984). Typicality and familiarity of faces. Memory \& Cognition, 12 (3), 219-228.doi: 10.3758/BF03197669

Benjamini, Y, \& Hochberg, Y. (1995). Controlling the false discovery rate: A practical and powerful approach to multiple testing. Journal of the Royal Statistical Society, Series B (57(1), 2890-300.

Bennetts, R. J., Kim, J., Burke, D., Brooks, K. R., Lucey, S., Saragih, J., Robbins, R. A. (2013). The movement advantage in famous and unfamiliar faces: a comparison of 
Motion Characteristics and Familiarity

point-light displays and shape-normalised avatar stimuli. Perception, 42, 850-970. doi:10.1068/p7446

Bernstein, M., \& Yovel, G. (2015). Two neural pathways of face processing: A critical evaluation of current models. Neuroscience and Biobehavioral Reviews, http://dx.doi.org/10.1016/j.neubiorev.2015.06.010

Bonner, L., Burton, M. A., \& Bruce, V. (2003). Getting to know your How we learn new faces. Visual Cognition, 10(5), 527-536. doi:10.1080/13506280244000168

Bruce, V., Henderson, Z., Greenwood, K., Hancock, P. J. B., Burton, A. M., \& Miller, P. (1999). Verification of face identities from images captured on video. Journal of Experimental Psychology-Applied, 5(4), 339-360. doi: 10.1037/1076-898X.5.4.339

Bruce, V., Henderson, Z., Newman, C., \& Burton, A. M. (2001). Matching identities of familiar and unfamiliar faces caught on CCTV images. Journal of Experimental Psychology-Applied, 7(3), 207-218. doi: 10.1037/1076-898X.7.3.207

Bruce, V., \& Young, A. (1998). In the eye of the beholder: the science of face perception. Oxford University Press.

Bülthoff, I., \& Newell, F. N. (2015). Distinctive voices enhance the visual recognition of unfamiliar faces. Cognition, 137, 9-12. doi:10.1016/j.cognition.2014.12.006 
Motion Characteristics and Familiarity

Burton, A. M, Wilson, S., Cowan, M \& Bruce, V. (1999). Face recognition in poor quality video: evidence from security surveillance. Psychological Science, 10, 243-248. doi: $10.1111 / 1467-9280.00144$

Butcher, N., Lander, K., Fang, H. \& Costen, N. (2011). The effect of motiour at encoding and retrieval for same and other race face recognition. British Journal of Psychology, 102, 931-942. doi: 10.1111/j.2044-8295.2011.02060.x

Cavanagh, P., \& Leclerc, Y. (1989). Shape from shadows. Journal of Experimental Psychology: Human Perception and Performance 15, 3-27. doi: 10.1037/00961523.15.1.3

Christie, F., \& Bruce, V. (1998). The role of dynamic information in the recognition of unfamiliar faces-Memory \& Cognition, 26(4), 780-790. doi: 10.3758/BF03211397

Cohen, J.D., MacWhinney, B., Flatt, M., \& Provost, J. (1993). PsyScope: A new graphic interactive environment for designing psychology experiments. Behavioural Research Methods, Instruments \& Computers, 25(2), 257-271.

Cristinacce, D., \& Cootes, T. F. (2008). Facial Motion Analysis using Clustered Shortest Path Tree Registration, Proc. MLVMA Workshop (ECCV). 
Motion Characteristics and Familiarity

Ellis, H. D., Shepherd, J. W., \& Davis, G. M. (1979). Identification of familiar and unfamiliar faces from internal and external features: some implications for theories of face recognition. Perception, 8, 431-439. doi: 10.1068/p080431

Galper, R. E. (1970). Recognition of faces in photographic negative, Psychonomic Science, 19, 207-208. doi: 10.3758/BF03328777

Hill, H., \& Bruce, V. (1996). The effects of lighting on the perception of facial surfaces. Journal of Experimental Psychology: Human Perception and Performance, 22, 9861004. doi:10.1037/0096-1523.22.4.986

Johnston, A., Hill, H. \& Carman, N. (1992). Recognising faces: Effects of lighting direction, inversion and brightness reversal. Perception, 21, 356-376. doi: 10.1068/p210365n

Kemp, R. Pike,G., White P. \& Musselman, A. (1996). Perception and recognition of normal and negative faces: the role of shape from shading and pigmentation cues. Perception, 25(1), 37-52.

Knappmeyer, B., Thornton, I., Bülthoff, H. (2003). The use of facial motion and facial form during the processing of identity. Vision Research, 43, 1921-1936. 
Motion Characteristics and Familiarity

Knight, B., \& Johnston, A. (1997). The role of movement in face recognition. Visual Cognition, 4(3), 265-273. doi: 10.1080/713756764

Lander, K., \& Bruce, V. (2000). Recognizing famous faces: Exploring the benefits of facial motion. Ecological Psychology, 12(4), 259-272.

Lander, K., \& Bruce, V. (2003). The role of motion in learning new faces. Visual Cognition, 10(8), 897-912. doi: 10.1207/S15326969ECO1204_01

Lander, K., Bruce, V., \& Hill, H. (2001). Evaluating the effectiveness of pixelation and blurring on masking the identity of familiar faces. Journal of Applied Cognitive Psychology, 15(1), 116. do1:10.1002/1099-0720(200101/02)

Lander, K., Christie, F., \& Bruce, V. (1999). The role of movement in the recognition of famous faces. Memory \& Cognition, 27(6), 974-985.

Lander, K. \& Chuang, L. (2005). Why are moving faces easier to recognize? Visual Cognition, 12(3), 429-442. doi: 10.3758/BF03201228 
Motion Characteristics and Familiarity

Lander, K. Chuang, L., \& Wickham, L. (2006). Recognizing face identity from natural and morphed smiles. The Quarterly Journal of Experimental Psychology, 59(5), 801-808. doi: $10.1080 / 17470210600576136$

Lander, K. \& Davies, R. (2007). Exploring the role of characteristic motion when learning new faces. The Quarterly Journal of Experimental Psychology, 60(4). 519-526. doi $10.1080 / 17470210601117559$

Light, L. L., Kayra-Stuart, F., \& Hollander, S. (1979) Recognition memory for typical ad unusual faces. Journal of Experimental Psychology: Human Learning and Memory 5(3), 212-228. doi: $10.1037 / 0278-7393 \cdot 5 \cdot 3.212$

Liu, C. H. \& Chaudhuri, A. (1998). Are there qualitative differences between face processing in photographic positive and negative? Perception, 27(9), 1107-22

Luria, S. M., \& Strauss, M. S. (1978). Comparison of eye movements over faces in photographic positives and negatives. Perception 7(3), $349-358$. 
Motion Characteristics and Familiarity

Morrison D J, Schyns P G. (2001). Usage of spatial scales for the categorization of faces, objects, and scenes. Psychonomic Bulletin \& Review. 8, 454-469. doi: $10.3758 / \mathrm{BF} 03196180$

Oliva, A., \& Schyns, P. G. (1997). Coarse blobs or fine edges? Evidence that information diagnosticity changes the perception of complex visual stimuli. Cognitive Psychology, 34, 72-107.

O'Toole, A. J., Roark, D. A., \& Abdi, H. (2002). Recognizing moving faces: a psychological and neural synthesis. Trends in Cognitive Sciences, 6(6), 261-266. doi:10.1016/S13646613(02)01908-3

Phillips, R. J. (1972). Why are faces hard to recognize in photographic negative. Perception \& Psychophysics, 12, 425-426. doi: 10.3758/BF03205854

Pike, G. E., Kemp,R I., Towell, N. A., \& Phillips, K. C. (1997). Recognizing Moving Faces: The Relative Contribution of Motion and Perspective View Information. Visual Cognition, 4(4), 409-438. doi: 10.1080/713756769

Roark, D. A., Barrett, S. E., Spence, M., Abdi, H., O'Toole, A. J. (2003). Memory for moving faces: Psychological and neural perspectives on the role of motion in face recognition. Behavioural and Cognitive Neuroscience Reviews, 2(1), 15-46. 
Motion Characteristics and Familiarity

Roark, D. A., O'Toole, A. J., Abdi, H., Barrett., S. E., (2006). Learning the moves: The effect of familiarity and facial motion on person recognition across large changes in viewing format. Perception, 35, 761-773.

Russell, R., Sinha, P., Biederman, I., \& Nederhouser, M. (2006). Is pigmentation important for face recognition? Evidence from contrast negation, Percepfion 35(6), 749-759.

Schiff, W., Banka, L., \& de Bordes Galdi, G. (1986). Recognizing people seen in events via dynamic "mug shots". The American Sournal of Psychology, 99(2), 219-231.

Schultz, J., Brockhaus, M., Bülthoff, H. H., \& Pilz, K. S. (2013). What the human brain likes about facial motion. Cerebral Cortex, 23, 1167-1178. doi: 10.1093/cercor/bhs106

Schyns, P. G., \& Oliva, A. (1999). Dr. Angry and Mr. Smile: When categorization flexibly modify the perception of faces in rapid visual presentation. Cognition, 69, 243-265. doi:10.1016/S0010-0277(98)00069-9

Thornton, I. M. \& Kourtzi, Z. (2002). A matching advantage for dynamic human faces. Perception, 31, 113-132. 
Motion Characteristics and Familiarity

Valentine, T., \& Bruce, V. (1986). The effects of distinctiveness in recognising and classifying faces. Perception, 15, 525-535.

Valentine, T. \& Ferrara, A. (1991). Typicality in categorization, recognition and identification: Evidence from face recognition. British Journal of Psychology, 82, 87102. doi: 10.1111/j.2044-8295.1991.tb02384.x

Vokey, J. R., \& Read, J. D. (1992). Familiarity, memorability, and the effect of typicality on the recognition of faces. Memory \& Cognition, 20, 291-302. doi: 10.3758/BF03199666

Xiao, N. G., Perrorra, S., Quinn, P. C., Wang, Z., Sun, Y. H. P., \& Lee, K. (2014). On the facilitative effects of face motion on face recognition and its development. Frontiers in Psychology, 5, 633. doi: 10.3389/fpsyg.2014.00633

Young, A. W., Hay, D.C McWeeny, K. H., Flude, B. M., \& Ellis, A. W. (1985). Matching familiar and unfamiliar faces on internal and external features. Perception, 14, 737-746.

\section{Figure Captions}

Figure 1. Scatter plots displaying the range of ratings of (a) familiarity, (b) amount of motion and (c) distinctiveness across the 58 faces (out of 10). 
Motion Characteristics and Familiarity

(a)

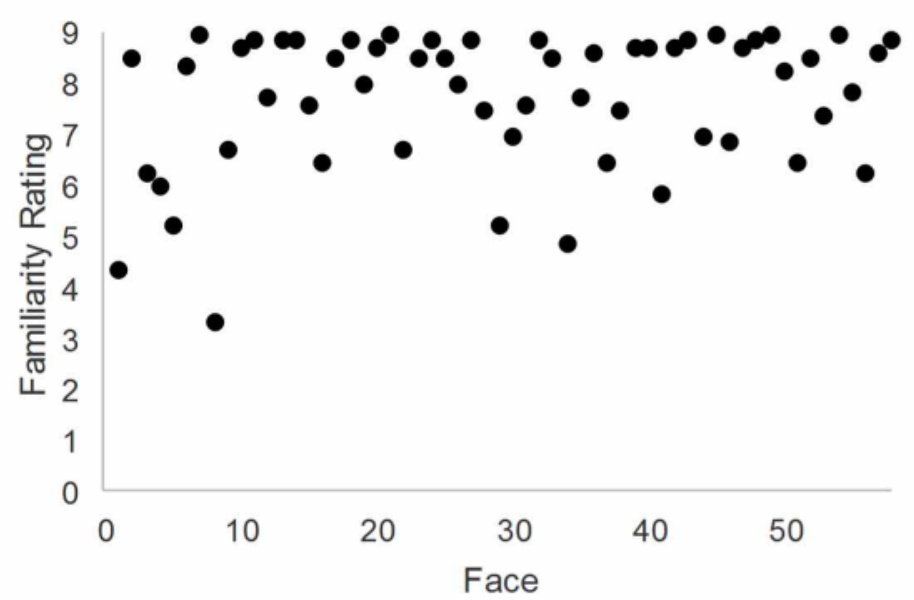

(b)

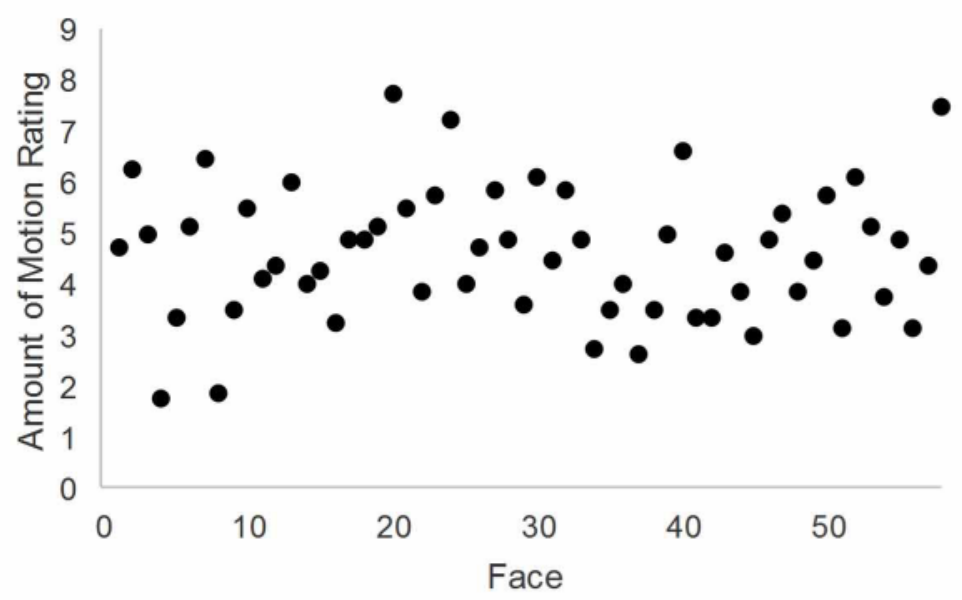

(c)

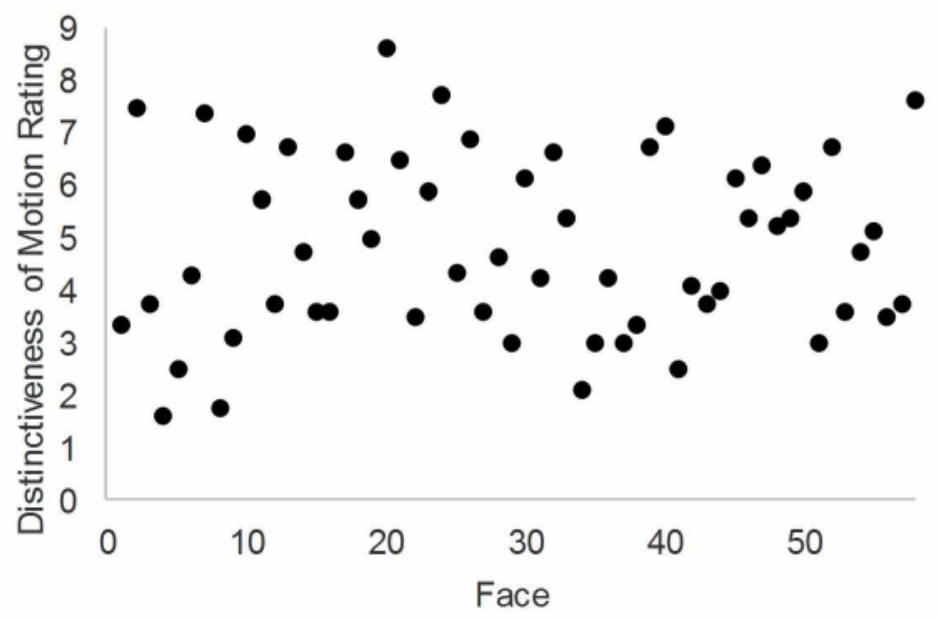

